\title{
ASTROPHYSICAL PLASMAS AND ATOMIC PROCESSES
}

\author{
J.S. KAASTRA \\ SRON \\ Sorbonnelaan2, 3584 CA Utrecht, The Netherlands
}

\section{Introduction}

Several plasma codes are available for the analysis of hot astrophysical plasmas. Among the oldest are the Raymond-Smith model (Raymond \& Smith 1977) and Mewe-Gronenschild model (Mewe et al. 1985, 1986). Minor updates to this last code have resulted in the meka model (Kaastra 1992); major updates (most importantly the ionization balance and the treatment of the Fe-L complex) resulted in the mekal code (Mewe, Kaastra \& Liedahl 1995). Here also the plasma codes of Masai (1984) and Landini \& Monsignori Fossi (1990) should be mentioned. The RS, meka and mekal codes are included in the XSPEC fitting package, the latest mekal code is incorporated in the SPEX package (Kaastra et al. 1996). Both Masai's code and SPEX contain non-equilibrium ionization (NEI) modes. All these codes differ in details, see Brickhouse et al. (1995) for an overview. Most important for the analysis of X-ray data is the ionization balance that is used for iron and the treatment of the Fe-L complex.

Masai (1997) has shown the role of the ionization balance. A simulated ASCA spectrum yielded best-fit temperatures of $0.85,1$ and $1 \mathrm{keV}$ and iron abundances of $0.7,0.9$ and 1.0 times solar using the ionization balance for iron of Arnaud \& Rothenflug (1985), Arnaud \& Raymond (1992) and Masai, respectively. Thus, the use of different ionization balances may lead to rather large differences in the derived parameters.

In a similar way, the treatment of the Fe- $\mathrm{L}$ complex can give large differences. For example, Matsushita (this conference) has fitted the spectrum of the elliptical galaxy NGC 4636 with different plasma codes. Comparing the RS and mekal codes, she finds for O-S abundances of 0.42 and 0.73 times solar, respectively, and for iron of 0.44 and 0.70 . The temperatures derived from both codes is 0.76 and $0.66 \mathrm{keV}$, respectively. In general, we 
Si $X N$

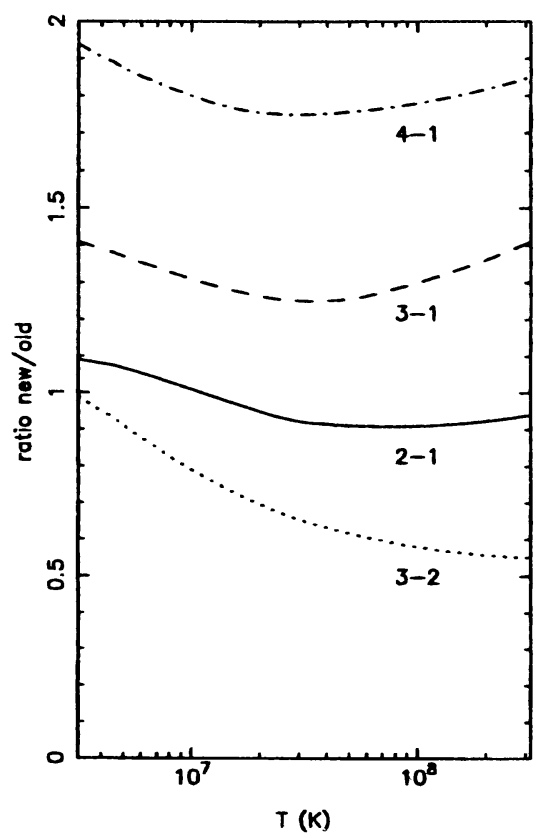

Fe $X X M$

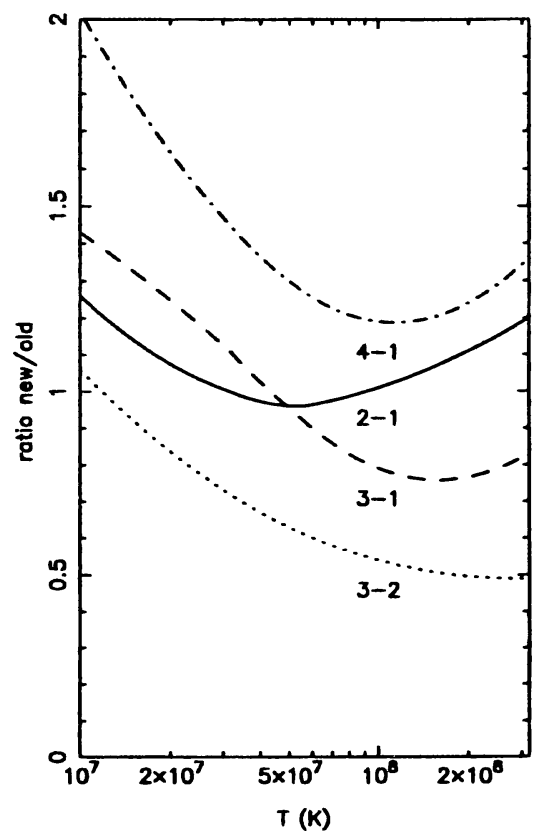

Figure 1. Ratio of new line calculations over old calculations as described in the text. Transitions shown are for Si XIV: 2-1 $(2.00 \mathrm{keV}), 3-1(2.38 \mathrm{keV}), 4-1(2.50 \mathrm{keV})$ and 3-2 (0.37 keV); for Fe XXVI: 2-1 (6.97 keV), 3-1 (8.25 keV), 4-1 (8.70 keV) and 3-2 (1.29 keV)

recommend presently the mekal code for the analysis of hot plasmas, although there are certainly still large uncertainties in several aspects of that code, as we show below.

\section{Excitation rates}

Triggered by the major improvements obtained for the Fe-L complex by using the latest excitation calculations available, and also by the need of higher accuracy and more details for the analysis of coming high-resolution missions such as AXAF, XMM and ASTRO-E, we (R. Mewe, J.S. Kaastra et al.) have started a project to revise and extend all atomic data used in the mekal-code. This includes a systematic search through the literature and a critical evaluation of the data used. Recently we finished the hydrogen iso-electronic sequence. Although it is generally assumed that the data for the $\mathrm{H}$ - and He-sequence are rather accurate, our investigation showed that this holds only for the 2-1 transitions in the H-sequence. We have used the most recent atomic data available for the excitation cross sections in order to determine the line strength for all transitions up to $n=5$ in 
Ne IX

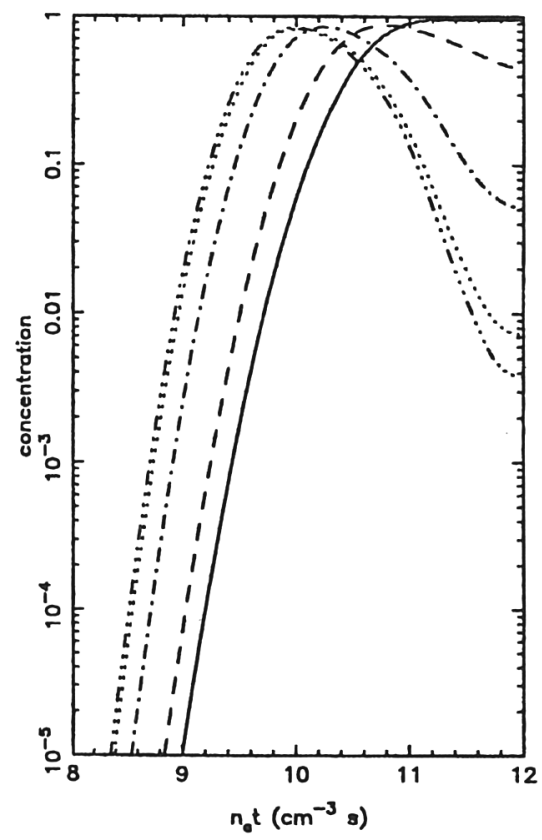

Ne $X$

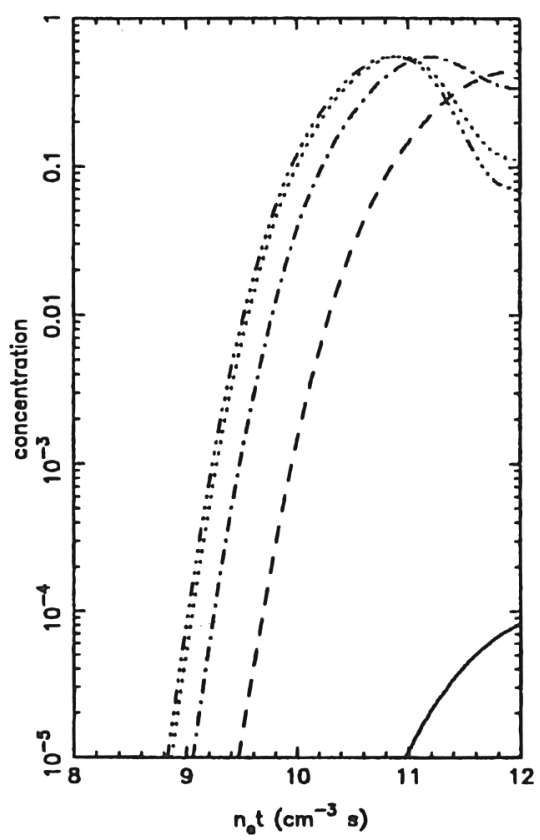

Figure 2. Ion fraction as a function of $n_{e} t$ for $\mathrm{Ne} \mathrm{IX}$ and $\mathrm{Ne} \mathrm{X}$ in an ionizing plasma with a temperature of $1 \mathrm{keV}$ with an additional cool electron component with a temperature $0.1 \mathrm{keV}$ and a relative density as compared to the $1 \mathrm{keV}$ component of $0(-\ldots), 0.1(\ldots), 1$ $(--), 9(-)$ and $\infty$ (solid line).

the H-sequence. These data include the effects of resonances at low energies and temperatures. In fig. 1 we plot the ratio of some of these newly calculated line strengths to the line strengths used in the mekal code. The data for Si XIV are from Aggarwal \& Kingston (1992) and for Fe XXVI from Kisielius et al. (1996). It is seen that for the most important temperature ranges the Lyman $\alpha(2-1)$ transitions are accurate to $\sim 10 \%$, but for Lyman $\beta$ (3-1) and Lyman $\gamma(4-1)$ the differences between the old and new calculations can be up to 40-70\%. A similar difference exists for $\mathrm{H} \alpha(3-2)$. It is evident that it is very important to have the best available rates for these ions in the plasma codes used for X-ray spectral modelling, and we intend to release an update of the SPEX code when the most important rates (including the $\mathrm{He}$ - and $\mathrm{Li}$-sequence and others) have been included.

\section{Non-equilibrium ionization}

Non-equilibrium ionization (NEI) plays a dominant role in supernova remnants. In most cases spectral fits are made based upon the assumption of a 
plasma that is instanteneously heated to the shock temperature and ionizes subsequently by collisions with thermal electrons. A Maxwellian distribution is not always the best description of the plasma, however. For example, Itoh (1984) showed that in the early stages of ionization $\left(n_{\mathrm{e}} t<310^{9} \mathrm{~cm}^{-3} \mathrm{~s}\right)$ the electron population consists of hot shock-heated electrons and cooler electrons ejected during the ionization process. He showed the effects of this on the plasma temperature evolution. However it also affects the NEI ionization process, as shown here. An additional cooler electron component has insufficient energy to ionize but gives mainly rise to an enhanced recombination rate, thereby slowing down the ionization process. This is illustrated in fig. 2 for $\mathrm{H}$-like and $\mathrm{He}$-like $\mathrm{Ne}$. As a result, the ionization time scale $t$ as derived from the measured ion concentrations is over-estimated by a factor which in the worst case can be an order of magnitude. It is evident that also the abundance estimates depend upon the presence or absence of such an additional cool electron component. An example where this process may play a role is RCW 86 (Vink et al 1997). ASCA spectra of this supernova remnant taken at different locii showed $n_{\mathrm{e}} t$ values of $\sim 210^{9} \mathrm{~cm}^{-3} \mathrm{~s}$, which combined with an electron density of $0.2 \mathrm{~cm}^{-3}$ leads to a typical plasma age of 300 year, much smaller than the age of the remnant (at least 2000 year). Moreover, the remnant shows very low abundances, not only for elements like $\mathrm{O}, \mathrm{Si}$ and $\mathrm{Fe}$, but also for $\mathrm{Ne}$, which is hard to reconcile with e.g. dust sputtering models, since Ne cannot be bound in dust. Given also the low $n_{\mathrm{e}} t$ value, Vink et al suggested that the above mentioned effect plays a role in RCW 86.

\section{References}

Aggarwal, K.M., Kingston, A.E. (1992): Phys.Scr., 46, 193

Arnaud, M., Raymond, J. (1992): ApJ 398, 394

Arnaud, M., Rothenflug, R. (1985): A\&AS 60, 425

Brickhouse, N., Edgar, R., Kaastra, J. et al (1995): Legacy 6, 4

Itoh, H. (1984): ApJ 285, 601

Kaastra, J.S. (1992): An X-ray spectral code for optically thin plasmas, Internal SRONLeiden report, version 2.0.

Kaastra, J.S., Mewe, R., Nieuwenhuijzen, H. (1996): in UV and X-ray spectroscopy of astrophysical and laboratory plasmas, p. 411, eds. K. Yamashita and T. Watanabe, Univ. Acad. Press

Kisielius, R., Berrington, K.A., Norrington, P.H. (1996): A\&AS 118, 157

Landini, M., Monsignori Fossi, B.C. (1990): A\&AS 82, 229

Masai, K. (1984): Ap\&SS 98, 367

Masai, K. (1997): A\&A 324, 410

Mewe, R., Gronenschild, E.H.B.M., Van den Oord, G.H.J. (1985): A\&AS 62, 197

Mewe, R., Kaastra, J.S., Liedahl, D.A. (1995): Legacy 6, 16

Mewe, R., Lemen, J.R., Van den Oord, G.H.J. (1986): A\&AS 65, 511

Raymond, J.C., Smith, B.W. (1977): ApJS 35, 419

Vink, J., Kaastra, J.S., Bleeker, J.A.M. (1997): A\&A, in press. 